\title{
Reply to Hochstenbach et al
}

\author{
European Journal of Human Genetics (2006) 14, \\ 1063-1064. doi:10.1038/sj.ejhg.5201663; published \\ online 31 May 2006
}

The introduction of the term 'molecular karyotyping' was a conscious decision, being well aware of both its limitations and advantages. Hochstenbach et al ${ }^{1}$ summon the shortcomings of this new term in genetics. Here, we would like to summarize the advantages of using this terminology and pinpoint some shortcomings in their reasoning.

The term karyotype was introduced by Levitsky. At that time, it was meant to describe nuclear morphology (as its Greek meaning explains). In 1931, Levitsky² wrote 'Me personally, used the term karyotype to define the characteristics of the nuclei from one or a group of organisms.' As in plant nuclei chromosomes were easily visualized, the karyotyping or looking at nuclei became synonymous. Because of this, the term has taken on its current definition as describing the particular chromosome complement of an individual, as defined by the number and morphology of the chromosomes. Thus, the meaning of the word 'karyotype' has evolved and is time and context dependent.

Karyotyping was the first genome-wide screening tool for chromosomal imbalances and - in addition - enables the identification of chromosomal translocations. Cytogenetics is the discipline in genetics that studies chromosomes and analyses karyotypes and most cytogeneticists, especially human cytogeneticists, are experts in karyotyping. In analogy with karyotyping, genome-wide array comparative genomic hybridization (CGH) or hybridization of singlenucleotide polymorphism (SNP) arrays enables the detection of genomic imbalances, albeit cannot, as of yet, detect chromosomal translocations. On the other hand, conventional karyotyping alone often shows aberrations that cannot be assigned to a chromosomal band and is not able to detect small aberrations or structural exchanges of similar banding pattern. 'Molecular karyotyping' is therefore the logical term for the different new molecular techniques for genome-wide profiling of chromosomal aberrations and places the technology used for these purposes within the realm of the expertise of cytogeneticists.

Rather than giving a new meaning to the word karyotype, a new word or expression 'molecular karyotyping' was coined. This word is not the same, nor meant to be the same as 'karyotype'. Its meaning is: 'A genome-wide intensity ratio profile comparing patient versus reference DNA'. An overview of genomic imbalances is derived using DNA from a mixture of cells in all stages of the cell cycles without a view of chromosome morphology. The term karyotype is used here as a metaphor, and the combination of molecular and karyotype creates a novel expression. The most important aspect of a language is that communities of people understand the same when using certain words. The genetics community is such an entity and it is our feeling that 'molecular karyotyping' better captures the spirit of the technique as compared with the introduction of yet another novel term 'segmental aneuploidy profiling'.

Moreover, genome-wide array CGH or hybridization of SNP arrays are not restricted to 'segmental aneuploidy', the new term proposed by Hochstenbach et al. ${ }^{1}$ The technique is also capable of showing whole chromosome aneuploidies. Hochstenbach et ll $^{1}$ argue that the term molecular karyotyping is already in use to indicate a flow cytometric karyotype. As flow cytometry is not implemented in medical genetic laboratories, the term does not create confusion in our discipline.

In conclusion, 'molecular karyotyping' is a simple term that was independently introduced by at least three groups. ${ }^{3-5}$ Hence, the term seems to both have the backing and meet the needs of the medical genetics community.

\section{Acknowledgements}

We thank Irina Balikova for the English translation from the original Russian article written by Levitsky.

Joris Robert Vermeesch ${ }^{*, 1}$ and Anita Rauch ${ }^{2}$

${ }^{1}$ Centre for Human Genetics, University of Leuven, University Hospital Gasthuisberg, Leuven, Belgium;

${ }^{2}$ Institute of Human Genetics, Friedrich-Alexander University Erlangen-Nuremberg, Erlangen, Germany

*Correspondence: Dr JR Vermeesch, Centre for Human Genetics, University of Leuven, University Hospital Gasthuisberg, Herestraat 49, Leuven 3000, Belgium. Tel: + 3216 345941; Fax: + 3216346061 ;

E-mail: Joris.Vermeesch@uz.kuleuven.ac.be

\section{References}

1 Hochstenbach R, van Amstel HK, Poot M: Microarray-based genome investigation: molecular karyotyping or segmental aneuploidy profiling? Eur J Hum Genet 2006; 14: 262-265.

2 Levitsky GA: Morphology of the chromosomes and the notion of karyotype in the systematics. Bull Appl Gen Plant Breeding 1931; 27: $187-240$.

3 Larrabee PB, Johnson KL, Pestova E et al: Microarray analysis of cell-free fetal DNA in amniotic fluid: a prenatal molecular karyotype. Am J Hum Genet 2004; 75: 485-491. 
4 Rauch A, Ruschendorf F, Huang J et al: Molecular karyotyping using an SNP array for genomewide genotyping. J Med Genet 2004; 41: 916-922.

5 Vermeesch JR, Melotte C, Froyen G et al: Molecular karyotyping: array CGH quality criteria for constitutional genetic diagnosis. J Histochem Cytochem 2005; 53: 413-422.

\section{No evidence for association of a European-specific chromosome 17 inversion with multiple sclerosis}

European Journal of Human Genetics (2006) 14, 1064. doi:10.1038/sj.ejhg.5201665; published online 31 May 2006

Stefansson et $a l^{1}$ recently reported a large inversion polymorphism on chromosome 17q21 that defines two chromosome lineages ( $\mathrm{H} 1$ and $\mathrm{H} 2$ ) differing in the orientation of a $900-\mathrm{kb}$ segment. Their data indicate that these lineages diverged more than 3 million years ago and have not since recombined. The $\mathrm{H} 2$ lineage is rare in Africans, East Asians and indigenous American populations but occurs at a frequency of about $20 \%$ in Caucasian populations, where it seems to be undergoing positive selection.

This geographical pattern is congruent with the epidemiology of multiple sclerosis (MS), which is characterised by a high prevalence in European populations and those of European descent, and lower risks for other ethnic groups. ${ }^{2}$ Moreover, the inversion lies in a region of suggestive linkage with susceptibility to MS. ${ }^{3}$ The $900-\mathrm{kb}$ inverted fragment contains a number of genes including corticotrophin releasing hormone receptor (CRHR1), microtubuleassociated protein tau (MAPT), $N$-ethylmaleimide sensitive factor (NSF), saitohin, LOC284058 and intramembrane protease-5 (IMP5).

In order to examine a possible role of the chromosome 17 inversion polymorphism in genetic susceptibility to MS, we typed 937 UK trio families (an affected individual and both parents) for single nucleotide polymorphism (SNP) rs9468 using a TaqMan Genotyping Assay-on-Demand (C_7563752_10, Applied Biosystems). SNP rs9468 is in complete linkage disequilibrium with the inversion, with the $\mathrm{C}$ allele perfectly predicting the $\mathrm{H} 2$ lineage. ${ }^{1}$ Only 76 of the 937 families $(8 \%)$ included in this study were also part of the previously published linkage screen. ${ }^{3}$ There was no significant deviation from Hardy-Weinberg equilibrium and genotyping call rate was $98.5 \%$. No Mendelian errors were observed, nor were any inconsistencies among 166 samples typed in duplicate, indicating a genotyping error rate of $<0.01 \%$. In our study population, the H2 lineage had a frequency of $23.7 \%$ in 3698 independent parental chromosomes. We did not observe any transmission distortion $(P=0.617)$ to affected offspring using the TRANSMIT program. ${ }^{4}$

In conclusion, we have not found evidence that the inversion polymorphism on chromosome 17 , which is undergoing positive selection in Europeans, influences susceptibility to MS. This suggests that the modest evidence for linkage with MS seen on 17q results from the effects of other loci in this region.

\section{Acknowledgements}

This work was supported by the Wellcome Trust (grant 057097), the Multiple Sclerosis Society of the United States (grant RG3500-A-1) and the Multiple Sclerosis Society of Great Britain and Ireland (grant 730/ 02). AG is a Postdoctoral Fellow of the Research Foundation Flanders (FWO - Vlaanderen).

An Goris ${ }^{*, 1,2}$, Melanie Maranian ${ }^{1}$, Amie Walton $^{1}$, Tai Wai Yeo $^{1}$, Maria Ban ${ }^{1}$, Julia Gray ${ }^{1}$, Alastair Compston ${ }^{1}$ and Stephen Sawcer ${ }^{1}$

${ }^{1}$ Neurology unit, Department of Clinical Neurosciences, University of Cambridge, Cambridge, UK;

${ }^{2}$ Laboratory of Neuroimmunology, Section of Experimental Neurology, Katholieke Universiteit Leuven, Leuven, Belgium

${ }^{*}$ Correspondence: Dr An Goris, Neurology unit, Department of Clinical Neurosciences, Box 165, Addenbrooke's Hospital, Hills Road, Cambridge CB2 2QQ, UK. Tel: +44 12232172 22; Fax: + 441223336943 ; E-mail:ag441@medschl.cam.ac.uk

\section{References}

1 Stefansson H, Helgason A, Thorleifsson G et al: A common inversion under selection in Europeans. Nat Genet 2005; 37: 129-137.

2 Rosati G: The prevalence of multiple sclerosis in the world: an update. Neurol Sci 2001; 22: 117-139.

3 International Multiple Sclerosis Genetics Consortium (IMGSC): A high-density screen for linkage in multiple sclerosis. Am J Hum Genet 2005; 77: 454-467.

4 Clayton D: A generalization of the transmission/disequilibrium test for uncertain-haplotype transmission. Am J Hum Genet 1999; 65: $1170-1177$. 